\title{
IL-6-mediated migration of human metastatic melanoma cells is reduced by simvastatin treatment
}

\author{
Christine Wasinger ${ }^{1}$, Christoph Minichsdorfer ${ }^{2}$, Martin Hohenegger ${ }^{1 *}$ \\ From 18th Scientific Symposium of the Austrian Pharmacological Society (APHAR). Joint meeting with the \\ Croatian, Serbian and Slovenian Pharmacological Societies. \\ Graz, Austria. 20-21 September 2012
}

\section{Background}

In 1987 the HMG-CoA reductase inhibitors, statins, were first marketed and are now widely used as well-tolerated therapeutics for hypercholesterolemia. High interleukin 6 (IL-6) plasma levels in melanoma patients are linked to a higher tumour burden and reduced overall survival. We have recently shown that simvastatin triggers apoptosis in human metastatic melanoma cells which is associated with concentration-dependent changes in autocrine IL- 6 secretion. Here, we investigated IL- 6 signalling with respect to proliferation and migration in human metastatic melanoma cells under statin application.

\section{Methods}

For this approach, human metastatic melanoma cells (A375, 518a2) were used for quantification of surface expression of the IL-6 receptor (IL-6-R/gp130) and for cell cycle with FACS analysis. Additionally, migration assays were carried out with simvastatin and/or IL-6 administration over time.

\section{Results}

Increasing concentrations of simvastatin led to morphological changes and detachment of the melanoma cells. After reseeding of the detached cells, A375 cells had a normal cell cycle profile while 518a 2 cells underwent apoptosis resulting in cell death. Moreover, simvastatin treatment enhanced the surface expression of the IL-6-R and the gp130 subunit in a time- and concentration-dependent manner. Both cell lines responded to IL- 6 treatment with increased proliferation and migration which was inhibited by simvastatin.

\section{Conclusion}

We demonstrate that simvastatin up-regulates the IL-6 pathway on the level of the heteromeric IL- 6 receptor. Although increased IL-6 receptor expression would imply a stronger IL- 6 signal, this is not seen in the presence of simvastatin. A novel therapeutic concept for simvastatin may emerge from the suppression of the IL-6-mediated proliferation and migration in metastatic melanoma cells.

\section{Acknowledgements \\ This work was supported by the Herzfeldersche Familienstiftung and the Austrian Science Fund FWF (P-22385).}

\section{Author details}

${ }^{1}$ Institute of Pharmacology, Center for Physiology and Pharmacology, Medical University of Vienna, 1090 Vienna, Austria. ${ }^{2}$ Department of Oncology, Medical University of Vienna, 1090 Vienna, Austria.

Published: 17 September 2012

* Correspondence: martin.hohenegger@meduniwien.ac.at 